\title{
Image Mosaic Based On Fisheye Camera
}

\author{
Xue Leng \\ School of Photoelectric Engineering, Changchun University of Science and Technology, Changchun, \\ 130000, China \\ lengxue79@126.com
}

\begin{abstract}
One of the hotspots in the field of computer vision is binocular stereo vision, fisheye images with its ultra-wide viewing angle in the field of computer vision has been more and more popular. Image mosaic image with a spatial overlap region of the same scene at different times, different perspectives or different camera alignment, in order to obtain larger field of view and higher resolution images. In this paper, image preprocessing, image matching, image fusion complete fisheye Image mosaics.
\end{abstract}

Keywords: Fisheye; Stereo Vision; Image mosaics.

\section{Introduction}

Image mosaics technology is the number of copies have overlapping areas of the picture makes up a large high-resolution ultra-wide, seamless panoramic image technology. Panorama stitching technology has a wide range of applications, it has become a hot research in the field of virtual reality. Fisheye image forming wide viewing angle, the resulting panorama can be applied to greatly reduce the number of images.

\section{Image Mosaic Method}

\subsection{Pretreatment fisheye image}

After the fish-eye image front hemisphere scene is mapped onto a plane, fisheye images will have a serious distortion. Because of this distortion, fisheye stitching multiple images are very tedious task, involving a lot of calculations and image processing. Since fish-eye image field of view of 180 degrees or more, so a corresponding spherical image close to the spindle axis OZ hemisphere, shown in Figure 1:

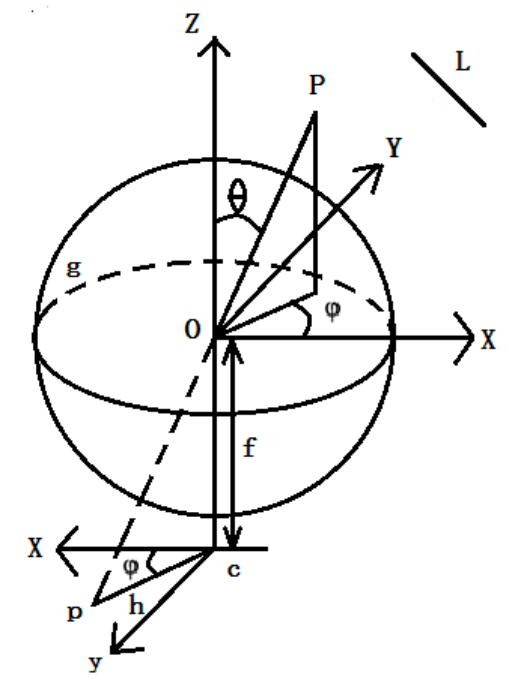

Fig1. Model of fisheye lens and the image plane Relations

If a point of spherical perspective projection point in the space is $\mathrm{P}$, Its spherical coordinates is $(\theta, \varphi)$, Fisheye distortion is $\mathrm{D}$, The point is mapped onto the image plane that fisheye is $\mathrm{n}$, the coordinate is $(x, y)$, it is the intersection of spindle and the fish-eye image plane. The main point coordinates is $\left(x_{p}, y_{p}\right)$. The main point as the coordinate origin of a polar coordinate system 
established polar coordinates, then the relationship between the Cartesian coordinate system and polar coordinate fisheye image point of the relationship are:

$$
\begin{aligned}
& r=\sqrt{\bar{x}^{2}+\bar{y}^{2}}, \operatorname{tg} \varphi=\frac{\bar{y}}{\bar{x}} \\
& x=x-x_{p}, y=y-y_{p}
\end{aligned}
$$

Suppose principal point is located in the center of the image, so fish-eye distortion correction model can be divided into tangential and radial distortion correction distortion correction. Tangential and radial distortion correction are as follows:

$$
\begin{aligned}
& \varphi=D_{T}^{-1}(\varphi) \\
& \theta=D_{R}^{-1}(r)
\end{aligned}
$$

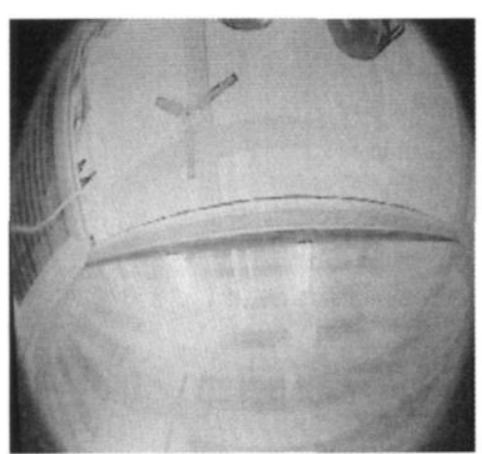

Fig2. Fisheye image before correction

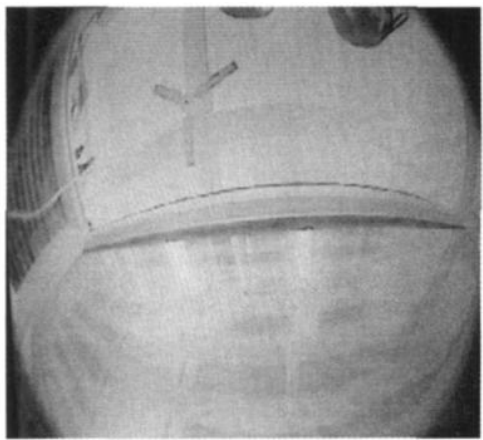

Fig3. Corrected fisheye image

Corrected fisheye image in line with people's intuitive sense, realistic strong, clear image edge.

\section{2 corner matching}

We can be seen from the two figures, the relatively large number of feature points, matching calculation capacity, directly affects the speed of image stitching. In order to improve the speed, in this paper, Image matching algorithm using cross-correlation. In the reference image and the image to be registered to each feature point serving as the center of a $(2 \mathrm{~N}+1) \times(2 \mathrm{~N}+1)$ gradation window, then the reference image of each feature point as a reference point to be image registration to find the corresponding match point, Matching is based on the calculated correlation coefficient between the feature points of the window.

$$
R=\frac{\left[\sum_{i=-N}^{N} \sum_{j=-N}^{N}(I(x-i, y-j)-\bar{l})\left(I^{\prime}\left(x^{\prime}-i, y^{\prime}-j\right)-\bar{I}^{\prime}\right)\right]}{\sqrt{\sum_{i=-N}^{N} \sum_{j=-N}^{N}\left(I(x-i, y-j)-I^{2}\right)^{2} \sum_{i=-N}^{N} \sum_{j=-N}^{N}\left(I^{\prime}\left(x^{\prime}-i, y^{\prime}-j\right)-I^{2}\right)^{2}}}
$$

$\bar{I}$ And $\bar{I}^{\prime}$ are the average brightness of all pixels in the gray scale window. Correlation values obtained by the formula $\mathrm{R}$, its threshold processing for 0.8 , for all $\mathrm{R}>0.8$ is regarded as a candidate corner points.

\subsection{Image Fusion}

The basic algorithm for linear weighted fusion is a simple way of weighting process, the positional relationship between the pixels in the overlapping area of the two images as weight information, so as to achieve the effect of a natural transition. For example, There are two pictures $I_{1}$ and $I_{2}$, They have the intersection area between the two pictures, and $I_{1}$ is in the left of $I_{2}$. Take any point $\mathrm{P}$ in the intersection area of pixels, If the point is at the center position of the intersection area, Then the weight values of $I_{1}$ is $50 \%$, the weight values of $I_{2}$ is $50 \%$.If $\mathrm{P}$ is the left-most point of the intersection area, Then the weight values of $I_{1}$ is $100 \%$, the weight values of $I_{2}$ is $0 \%$. This can be seen, the cross-regional integration is linear as linear aliquot area closer to the left of the image, the greater the weight of the left, while the right to decline the right weight. It can be expressed as: 
$P(x, y)=\frac{x-x_{s}}{x_{e}-x_{s}} I_{1}(x, y)+\frac{x_{e}-x}{x_{e}-x_{s}} I_{r}(x, y)$

$P(x, y)$ Is the gray value of $\mathrm{x}$ and $\mathrm{y} . x_{s}$ is the pixel coordinates of the starting point of the intersection region. $x_{e}$ Is the end region of the pixel coordinates of the intersection area? $I_{1}(x, y)$ And $I_{2}(x, y)$ indicate the direction in the image area of the left and right cross direction of the gray level change points. If it is a color image, it is respectively three-channel RGB images using this calculation method, respectively, in the last three color channels to calculate the final color values.

\subsection{Experiment results}

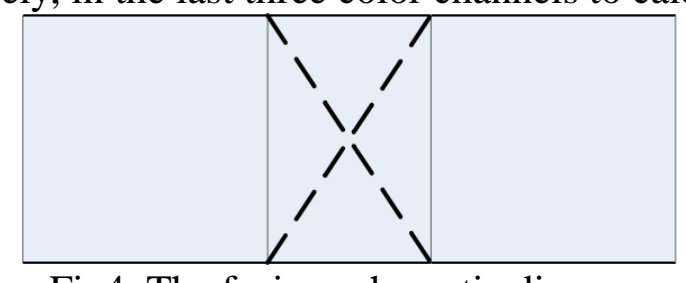

Fig4. The fusion schematic diagram

The resultings of image stitching and image fusion are as follows:

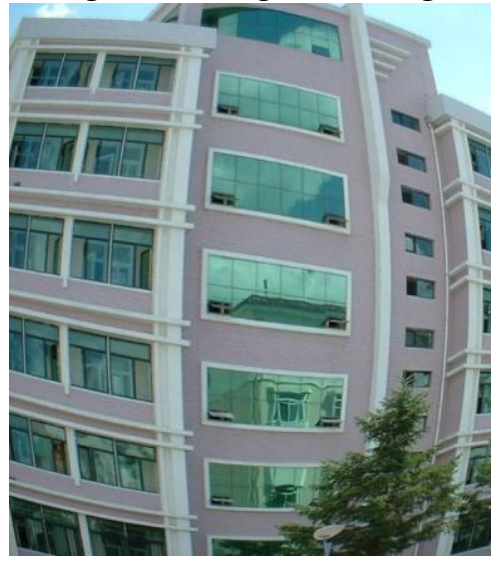

Fig5. The original image

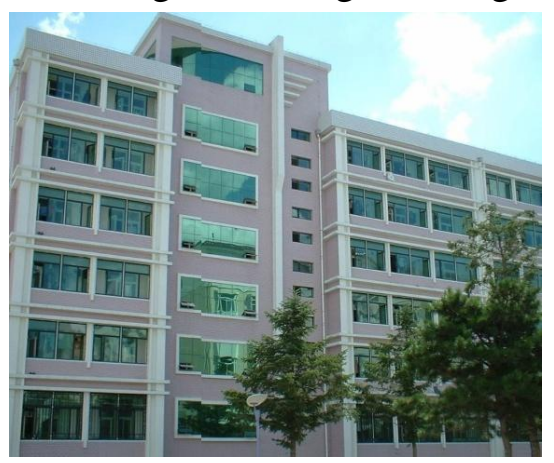

Fig7. Mosaiced images

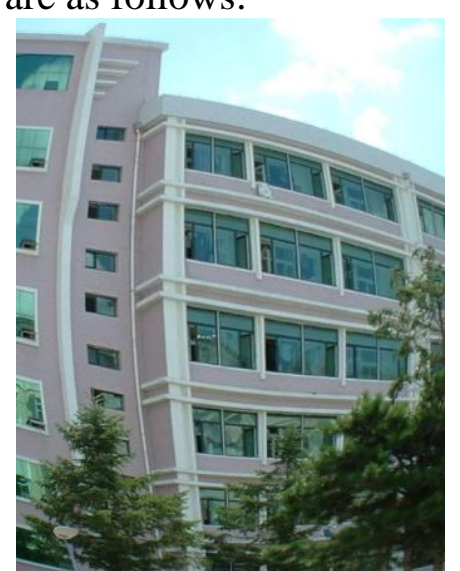

Fig6. Be mosaic image

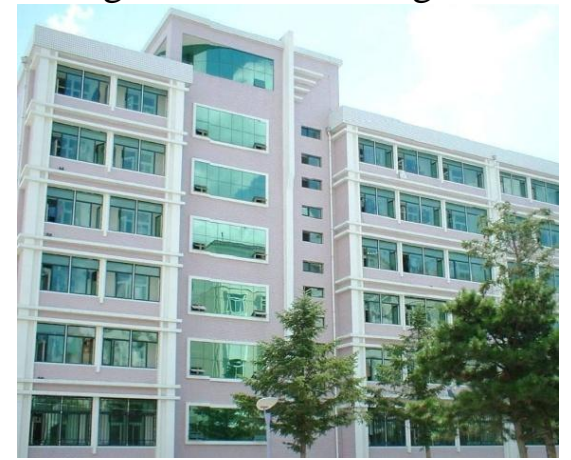

Fig8. Fused image

\section{Summary}

In this paper, the broad vision characteristic fisheye lens, a method of stitching fisheye images were studied. Bilinear interpolation method for correcting fisheye images using Image mosaics algorithm Harris feature points matching method and linear weighted fusion spliced image fusion process, simple algorithm can satisfy the real-time requirements .

\section{References}

[1]. Wei X. Fisheye thematic generalization. Digital Photography.Vol2 (2010) No20, P67-71. 
[2]. Jiesong D, Using of fisheye lens to generate a panoramic image method. Journal of Engineering Graphics. Vol15 (2010) No.1, P135-138.

[3]. Ping Gong, Xiangbin Liu, Peng Zhou. Corner Detection Based on an Improved Harris Algorithm. Vol.46 (2010) No11, p173-175.

[4]. Bo-Chao L.Improved Harris Corner Detection Method Based on Edge. Chinese Journal of Liquid Crystals and Displays, Vo128 (2013).No6, P939-942. 